TRANSACTIONS OF THE

AMERICAN MATHEMATICAL SOCIETY

Volume 196, 1974

\title{
AN INTERNAL CHARACTERIZATION OF PARACOMPACT $p$-SPACES
}

BY

\author{
R. A. STOLTENBERG ${ }^{(1)}$
}

ABSTRACT. The purpose of this paper is to characterize paracompact p-spaces in terms of spaces with refining sequences mod $k$. A space $X$ has a refining sequence $\bmod k$ if there exists a sequence $\left\{G_{n} \mid n \in N\right\}$ of open covers for $X$ such that $n_{n \geq 1}^{\infty} \mathrm{St}\left(C, \varrho_{n}\right)=P_{C}^{1}$ is compact for each compact subset $C$ of $X$ and $\left\{\mathrm{St}\left(C, \Theta_{n}\right)^{n \geq 1} \mid n \in N\right\}$ is a neighborhood base for $P_{C}^{1}$. If $P_{C}^{1}=C$ for each compact subset $C$ of $X$ then $X$ is metrizable. On the other hand if we restrict the set $C$ to the family of finite subsets of $X$ in the above definition then we have a characterization for strict p-spaces. Moreover, in this case, if $P_{C}^{1}=C$ for all such sets then $X$ is developable. Thus the concept of a refining sequence mod $k$ is natural and it is helpful in understanding paracompact p-spaces.

1. Introduction. In 1963 Arhangel'skii (see [2] and [4]) introduced the concept of a $p$-space. He defined a completely regular space $X$ to be a $p$-space if there exists a sequence $\left\{\mathcal{H}_{n} \mid n \in N\right\}$ of open collections in the Stone-Čech compactification of $X$ such that $\mathcal{H}_{n}$ covers $X$ for each $n \in N$ and such that, for each $x \in X, \bigcap_{n=1}^{\infty} \operatorname{St}\left(x, \mathcal{H}_{n}\right) \subseteq X$ where $\operatorname{St}\left(x, \mathcal{H}_{n}\right)=\bigcup\left\{H \mid x \in H\right.$ and $\left.H \in \mathcal{H}_{n}\right\}$. A sequence $\left\{\mathcal{H}_{n} \mid n \in N\right\}$ of open collections in the Stone-Čech compactification of $X$ is called a p-sequence if it satisfies the above conditions. Arhangel'skir characterized the class of perfect preimages of metric spaces to be the class of paracompact $p$-spaces which answered a question of Aleksandrov in [1].

There is another class of spaces that is related to the class of p-spaces under certain restrictions and that is the class of $M$-space. Morita [9] introduced the concept of an M-space in 1964. A topological space is an M-space if there exists a normal sequence $\left\{\mathcal{U}_{i} \mid i \in N\right\}$ of open covers of $X$ satisfying the condition (M):

Presented to the Society, January 22, 1971 under the title $A$ note on paracompact p-spaces; received by the editors May 31, 1972.

AMS (MOS) subject classifications (1970). Primary 54D20; Secondary 54D35.

Key words and phrases. Metric spaces, paracompact p-spaces, developable spaces, sequences of open covers, subparacompact spaces.

(1) This work was supported in part by a research grant from Sam Houston State University.

Copyright $\odot 1974$, American Mathematical Society 


$$
\left\{\begin{array}{l}
\text { If }\left\{K_{i} \mid i \in N\right\} \text { is a sequence of nonempty subsets of } X \text { such that } \\
K_{i+1} \subseteq K_{i}, K_{i} \subseteq S_{t}\left(x_{0} U_{i}\right) \text { for each } i \in N \text { and for some fixed point } \\
x_{0} \in X, \text { then } \bigcap_{i=1}^{\infty} K_{i}^{-} \neq \varnothing .
\end{array}\right.
$$

A sequence of covers $\left\{\mathcal{U}_{i} \mid i \in N\right\}$ is a normal sequence if and only if $\mathcal{U}_{i+1}$ star refines $\mathcal{U}_{i}$ for each $i$, that is, for each $U \in \mathcal{U}_{i+1}$ there exists $V \in \mathcal{U}_{i}$ such that St $\left(U, \mathcal{U}_{i+1}\right) \subseteq V$. Morita proved in [10] that the class of quasi perfect preimages of metric spaces is precisely the class of $M$-spaces. A map $f$ from a space $X$ onto a space $Y$ is quasi perfect if and only if it is closed continuous map such that $f^{-1}(y)$ is countably compact for each $y \in Y$. Thus a relationship between $M$-spaces and $p$-spaces is established.

1.1 Theorem. If $X$ is a completely regular metacompact space then $X$ is an M-space if and only if it is a paracompact p.space.

There is yet another approach to the concept of a paracompact $p$-space and it involves a property related to the concept of a development for a space. It is well known that a topological space $X$ is developable if and only if there exists a sequence $\left\{\varrho_{n} \mid n \in N\right\}$ of open covers of $X$ such that for each open set $U$ in $X$ and for each $x \in U$ there exists $n \in N$ such that $S_{t}\left(x, \Theta_{n}\right)=\bigcup\left\{G \in \mathscr{Q}_{n} \mid x \in G\right\} \subseteq U$. The sequence $\left\{\varrho_{n} \mid n \in N\right\}$ of open covers of $X$ is called a development for $X$. If the concept of a development is strengthened by replacing the point $x$ in the definition with a compact set $C$ one obtains Arhangel'skii's definition in [3] of a $k$-refining sequence, that is, a sequence of open covers $\left\{\varrho_{n} \mid n \in N\right\}$ is said to be a $k$-refining sequence if and only if for each compact set $C$ contained in $X$ and each open subset $U$ of $X$ containing $C$, there exists $n \in N$ such that $\operatorname{St}\left(C, \mathcal{G}_{n}\right) \subseteq U$. Arhangel'skir proved in [3] that spaces with this property are metrizable, that is:

1.2 Theorem. A regular topological space is metrizable if and only if it bas a k-refining sequence.

A concept is now introduced which is a natural generalization of the concept of a $k$-refining sequence and which will characterize paracompact $p$-spaces.

1.3 Definition.(2) A topological space $X$ is said to have a refining sequence

(2) For $T_{2}$ spaces it suffices to assume that $\left\{\mathrm{St}_{\mathrm{t}}\left(C, \varrho_{n}\right) \mid n \in N\right\}$ is a neighborhood base for $P_{C}^{1}$. Therefore in view of Lemma 2.1 we have that $\left\{\mathscr{Q}_{n} \mid n \in N\right\}$ is a refining sequence $\bmod k$ iff $\left\{\mathrm{St}_{\mathrm{t}}\left(C, \mathscr{S}_{n}\right) \mid n \in N\right\}$ is a $k$-sequence for each compact subset $C$ of $X$. The definition of $k$-sequence is given by $\mathrm{E}$. Michael in $A$ quintuple quotient quest, General Topology and Appl. 2 (1972), 91-138. 
$\bmod k$ if and only if there exists a sequence $\left\{\varrho_{n} \mid n \in N\right\}$ of open covers such that for each compact subset $C$ of $X$ the following hold:

(a) $\bigcap_{n=1}^{\infty}$ St $\left(C, \varrho_{n}\right)=P_{C}^{1}$ is compact.

(b) The collection $\left.\left\{\mathrm{St}_{\mathrm{t}}(C), \varrho_{n}\right)-\mid n \in N\right\}$ is neighborhood base for $P_{C}^{1}$.

The term refining sequence mod $k$ seems to be appropriate in light of the foregoing discussion and in view of the main result in this paper.

1.3 Theorem. A Hausdorff topological space $X$ is a paracompact p-space if and only if it has a refining, sequence $\bmod k$.

Thus it is established that the class of perfect preimages of metric spaces, the class of metacompact $M$-spaces, the class of paracompact $p$-spaces and the class of spaces with refining sequences $\bmod k$ are all the same. Moreover, because of the correlation between Theorems 1.2 and 1.3 the position in which paracompact $p$-spaces lie is a little more apparent.

In $\$ 2$ the basic properties of spaces with refining sequences $\bmod k$ are studied, and the other three sections depend on this section. In addition $\$ 4$ depends on the results in $\$ 3$ and $\$ 5$ depends on both $\$ \$ 3$ and 4. However, if one wants to assume the results in $\$ 3$ to be true, $\$ 4$ can be read without reading $\$ 3$. Similarly $\$ 5$ can be read without reading $\$ \$ 3$ and 4 .

Throughout this paper it will be assumed that all spaces are Hausdorff. The letter $N$ will denote the set of natural numbers and $\beta X$ will denote the Stone-Čech compactification of a completely regular space $X$. If $A$ is a subset of $X$ then $A^{-}$will denote the closure of $A$ in $X$ and $A_{\bar{B} X}^{-}$will denote the closure of $A$ in $\beta X$ in those cases where $\beta X$ is involved. $\Lambda$ base for the neighborbood system of the set $A$ is defined to be any collection of open sets with the property that if $V$ is an open set containing $A$, then there exists $U \in \mathcal{U}$ with $A \subseteq U \subseteq V$. If $X$ is a topological space with a refining sequence $\left\{\varrho_{n} \mid n \in N\right\} \bmod k$ then for any compact subset $C \subseteq X$ let $P_{C}^{1}=\bigcap_{n=1}^{\infty}$ St $\left(C, \Theta_{n}\right)$, and by induction let $P_{C}^{k+1}=$ $\bigcap_{n=1}^{\infty}$ St $\left(P_{C}^{k}, \varrho_{n}\right)$. In case $C=\{x\}$ for some $x \in X$ let $\left.P_{x}^{k}\right\}=P_{x}^{k}$ for each $k \in N$.

An attempt has been made to use standard notation and terminology. The principal reference used in this connection is [8].

2. General properties of spaces with refining sequence $\bmod k$. The properties developed in this section are basic to the study of spaces with refining sequences mod $k$. The notation and terminology introduced here will be used throughout the rest of this paper.

2.1 Lemma. If $X$ is a space with a refining sequence $\left\{\bigcup_{n} \mid n \in N\right\} \bmod k$ then there exists a refining sequence $\left\{\mathcal{H}_{n} \mid n \in N\right\} \bmod k$ for $X$ sucb that $\mathcal{H}_{n+1}$ refines $\mathcal{H}_{n}$ for each $n \in N$. 
Proof. Let $\mathcal{H}_{1}=\varrho_{1}, \mathcal{H}_{2}=\varrho_{1} \cap \varrho_{2}, \mathcal{H}_{3}=\varrho_{1} \cap \varrho_{2} \cap \varrho_{3}, \ldots, H_{n}=$ $\bigcap_{i=1}^{n} \Theta_{i}, \ldots$. We show that $\left\{\mathcal{H}_{n} \mid n \in N\right\}$ is the desired sequence. Clearly $\mathcal{H}_{n+1}$ refines $\mathcal{H}_{n}$ for each $n$. Let $C$ be a compact subset of $X$ and let $x \in P_{C}^{1}$ where $P_{C}^{1}=$ $\bigcap_{n=1}^{\infty} \operatorname{St}\left(C, \varrho_{n}\right)$; then $x \in \operatorname{St}\left(C, \varrho_{n}\right)$ for each $n \in N$. Thus $\bigcap_{i=1}^{\infty} \operatorname{St}\left(x, \varrho_{i}\right) \cap C \neq \varnothing$; for if $\bigcap_{i=1}^{\infty}$ St $\left(x, \varrho_{i}\right) \cap C=\varnothing$ then $\bigcap_{i=1}^{\infty} \operatorname{st}\left(x, \varrho_{i}\right)^{-} \cap C=\varnothing$ and thus there is $k \in N$ such that $\bigcap_{i=1}^{k}$ St $\left(x, \varrho_{i}\right)-\cap C=\varnothing$. Clearly $\bigcap_{i=1}^{k}$ St $\left(x, \varrho_{i}\right) \supseteq P_{x}^{1}$ and is an open set. So there exists $n \in N$ such that $P_{x}^{1} \subseteq S t\left(x, \varrho_{n}\right) \subseteq \bigcap_{i=1}^{k}$ St $\left(x, \varrho_{i}\right)$; but

$$
\varnothing \neq \operatorname{St}\left(x, \Theta_{n}\right) \cap C \subseteq \bigcap_{i=1}^{k} \operatorname{St}\left(x, \mathscr{S}_{i}\right) \cap C \subseteq \bigcap_{i=1}^{k} \operatorname{St}\left(x, \Theta_{i}\right)-\cap C,
$$

a contradiction. Let $y \in \bigcap_{i=1}^{k}$ St $\left(x, \varrho_{i}\right) \cap C$. Then for each $i \leq k$ there is $G_{i} \in \mathfrak{S}_{i}$ such that $x$ and $y \in G_{i}$. So $x$ and $y \in \bigcap_{i=1}^{k} G_{i} \in \mathcal{H}_{k}$, that is St $\left(x, \mathcal{H}_{k}\right) \cap C \neq \varnothing$ for any $k \in N$. So $x \in \mathrm{St}\left(C, \mathcal{H}_{k}\right)$ for each $k \in N$ or $x \in$ $\bigcap_{k=1}^{\infty} \mathrm{St}_{\mathrm{t}}\left(C, \mathcal{H}_{k}\right)$ and it follows that $P_{C}^{1} \subseteq \bigcap_{k=1}^{\infty} \mathrm{St}\left(C, \mathcal{H}_{k}\right)$. Clearly

$$
\bigcap_{k=1}^{\infty} \operatorname{St}\left(C, \mathcal{H}_{k}\right) \subseteq \bigcap_{n=1}^{\infty} \operatorname{St}\left(C, \mathcal{G}_{n}\right)=P_{C}^{1}
$$

So $P_{C}^{1}=\bigcap_{n=1}^{\infty}$ St $\left(C, \mathcal{H}_{n}\right)$. Since

$$
P_{C}^{1} \subseteq \operatorname{St}\left(C, \mathcal{H}_{n}\right) \subseteq \operatorname{St}\left(C, H_{n}\right)^{-} \subseteq \operatorname{St}\left(C, \Theta_{n}\right)^{-}
$$

it follows that $\left\{\mathrm{St}\left(C, \mathcal{H}_{n}\right)-\mid n \in N\right\}$ is a neighborhood base for $P_{C}^{1}$ and thus that $\left\{\mathcal{H}_{n} \mid n \in \dot{N}\right\}$ is a refining sequence $\bmod k$ for $X$.

From now on we will assume that a refining sequence $\left\{\varrho_{n} \mid n \in N\right\} \bmod k$ has the property that $\varrho_{n+1}$ refines $\varrho_{n}$ for each $n \in N$.

2.2 Lemma. If $X$ is a space with a refining sequence $\bmod k$ then $X$ is a regular space.

Proof. Let $\left\{\varrho_{n} \mid n \in N\right\}$ be a refining sequence $\bmod k$ for $X$; let $x \in X$ and let $B$ be a closed set such that $x \notin B$. Recall that $P_{x}^{1}=\bigcap_{n=1}^{\infty}$ St $\left(x, \Theta_{n}\right)$ is compact. So there exist disjoint open sets $U$ and $V$ such that $x \in U, V \supseteq B \cap$ $P_{x}^{1}$ since $X$ is $T_{2}$. Also there exists $n \in N$ such that $S_{t}\left(x, \varrho_{n}\right)-\cap B-V=\varnothing$ since $P_{x}^{1} \subseteq X-(B-V)$ and $X-(B-V)$ is an open set. Let $G$ be any member of $\varrho_{n}$ such that $x \in G$ and let $O=G \cap U$. Now $O$ is an open neighborhood of $x$ and $O^{-} \subseteq \dot{\mathrm{St}}\left(x, \varrho_{n}\right)^{-} \cap U^{-}$. So $O^{-} \cap B=\varnothing$ and thus $X$ is regular.

\subsection{Lemma. Let $X$ be a topological space with a refining sequence} $\left\{\varrho_{n} \mid n \in N\right\} \bmod k$ and let $C$ be any compact subset of $X$. If $\left\{x_{n} \mid n \in N\right\}$ is a sequence of points in $X$ such that $x_{n} \in S_{t}\left(C, \varrho_{n}\right)$ for each $n \in N$, then $\left\{x_{n} \mid n \in N\right\} \cup P_{C}^{1}$ is compact. Moreover every cluster point of the sequence $\left\{x_{n} \mid n \in N\right\}$ is in $P_{C}^{1}$. 
Proof. Suppose $A \subseteq\left\{x_{n} \mid n \in N\right\} \cup P_{C}^{1}$ and that $A$ has no limit points. Then $A \cap P_{C}^{1}$ is a finite set since $P_{C}^{1}$ is compact. Also $A-P_{C}^{1}$ is closed since $A$ has no limit points. Thus $X-\left(A-P_{C}^{1}\right)$ is an open set in $X$ containing $P_{C}^{1}$ and so there exists $n \in N$ such that $\operatorname{St}\left(C, \mathfrak{S}_{n}\right) \subseteq X-\left(A-P_{C}^{1}\right)$. Thus

$$
A-P_{C}^{1} \subseteq X-\operatorname{St}\left(C, \mathcal{G}_{n}\right) \subseteq X-\left\{x_{k} \mid k \geq n, k \in N\right\} .
$$

So $A-P_{C}^{1} \subseteq\left\{x_{k} \mid k \leq n\right\}$ and hence $A$ is a finite set. Therefore $\left\{x_{k} \mid k \in N\right\} \cup P_{C}^{1}$ is compact.

If $x$ is a cluster point of $\left\{x_{k} \mid k \in N\right\}$ then $x \in\left[S t\left(C, \varrho_{n}\right)\right]^{-}$for each $n_{\text {. }}$ Thus $x \in \bigcap_{n=1}^{\infty}\left[\mathrm{St}\left(C, \mathcal{G}_{n}\right)\right]^{-}=P_{C}^{1}$ and this completes the proof.

2.4 Lemma. Let $X$ be a topological space with a refining sequence $\left\{\varrho_{n} \mid n \in N\right\} \bmod k$, let $C$ be a compact subset of $X$ and let $\left\{x_{n} \mid n \in N\right\}$ be a sequence in $X$ such that $x_{n} \in \mathrm{St}\left(C, \mathcal{G}_{n}\right)$ for each $n \in N$. If $y_{k} \in \mathrm{St}\left(\left\{x_{i} \mid i \in N, i \geq k\right\}^{-}, \varrho_{k}\right)$ for each $k \in N$ then $\left(\left\{y_{k} \mid k \in N\right\}\right)^{-}$is compact and every cluster point of the sequence $\left\{y_{k} \mid k \in N\right\}$ is in $\bigcup\left\{P_{x}^{1} \mid x\right.$ is a cluster point of $\left.\left\{x_{n} \mid n \in N\right\}\right\}$.

The proof of the above lemma is similar to the proof of Lemma 2.3 and is omitted.

2.5 Lemma. Let $\left\{\varrho_{n} \mid n \in N\right\}$ be a refining sequence $\bmod k$ for $X$ and let $C$ be a compact subset of $X$. Then $P_{C}^{1}=\bigcup\left\{P_{z}^{1} \mid z \in C\right\}$.

Proof. For each $n$,

$$
\operatorname{st}\left(C, \varrho_{n}\right)=\bigcup\left\{G \in \mathscr{O}_{n} \mid G \cap C \neq \varnothing\right\}=\bigcup\left\{\operatorname{St}\left(z, \bigodot_{n}\right) \mid z \in C\right\} \supseteq \bigcup\left\{P_{z}^{1} \mid z \in C\right\}_{0}
$$

Thus $P_{C}^{1} \supseteq\left\{P_{z}^{1} \mid z \in C\right\}$.

Conversely, suppose $x \in P_{C}^{1}$ then for each $n \in N$ there exists $x_{n} \in C$ such that $x \in \mathrm{St}\left(x_{n}, \varrho_{n}\right)$. Thus $x_{n} \in \mathrm{St}\left(x, \varrho_{n}\right)$ for each $n \in N$ and thus $\left\{x_{n} \mid n \in N\right\}$ has a cluster point $y$ in $P_{x}^{1}$. Now recall that $x_{n} \in C$ for each $n$; so $y \in C$. Moreover if $y \in P_{x}^{1}$ it follows that $x \in P_{y}^{1}$ and so $x \in \bigcup\left\{P_{z}^{1} \mid z \in C\right\}$. Thus $P_{C}^{1}=\bigcup\left\{P_{z}^{1} \mid z \in C\right\}$.

Recall that in the introduction we let $P_{x}^{1}=\bigcap_{n=1}^{\infty}$ St $\left(x, \Theta_{n}\right), P_{x}^{2}=$ $\bigcap_{n=1}^{\infty}$ St $\left(P_{x}^{1}, \varrho_{n}\right)$ and so on. It follows from Lemma 2.5 that $P_{x}^{x}=$ $\bigcup\left\{P_{w}^{1} \mid w \in P_{x}^{k-1}\right\}$ for $k \geq 2$. Thus for each $k \geq 2 z \in P_{x}^{k}$ if and only if there exists a finite sequence $z_{0}, z_{1}, \ldots, z_{k}$ such that $z_{0}=x_{0} z_{1} \in P_{z_{0}}^{1}, z_{2} \in P_{z_{1}}^{1^{\circ}}$, $\cdots, z_{k} \in P_{z_{k-1}}^{1}$ and $z_{k}=z$.

2.6 Lemma. For each $x \in X$ let $P_{x}^{\omega}=\bigcap_{n=1}^{\infty} P_{x^{*}}^{n}$ Then $\left\{P_{x}^{\omega} \mid x \in X\right\}$ partition $X$.

Proof. Suppose $x, y \in X$ and $P_{x}^{\omega} \cap P_{y}^{\omega} \neq \varnothing$. Let $z \in P_{x}^{\omega} \cap P_{y}^{\omega}$. Then $z \in P_{x}^{k}$, 
and $z \in P_{y}^{m}$ for some $k$ and $m \in N$. Now $P_{y}^{m+1}=\bigcup\left\{P_{w}^{1} \mid w \in P_{y}^{m}\right\}$. So $P_{z}^{1} \subseteq P_{y}^{m+1}, P_{z}^{2} \subseteq P_{y}^{m+2}, \ldots, P_{z}^{i} \subseteq P_{y}^{m+i}, \ldots$. If $w \in P_{x}^{w}$ then $w \in P_{x}^{n}$ for some $n \in N$ and there exist $w_{0}, \ldots, w_{n}$ such that $w_{i+1} \in P_{w_{i}}^{1}$ for $i=0,1,2, \ldots$, $n-1$ where $w_{0}=x$ and $w_{n}=w$. Also there exist $z_{0}, z_{1}, \ldots, z_{k}$ such that $z_{i+1} \in P_{z_{i}}^{1}$ for $i=0,1,2, \cdots, n$ where $z=z_{n}$ and $x=z_{0}$. Hence it follows that $w \in P_{z}^{n+k} \subseteq P_{y}^{m+n+k} \subseteq P_{y^{*}}^{\omega}$ Thus $P_{x}^{\omega} \subseteq P_{y^{*}}^{\omega}$ By a similar argument it can be shown that $P_{y}^{\omega} \subseteq P_{x}^{\omega}$ and thus the lemma follows.

Let $\Gamma$ be the first ordinal number whose cardinal number equals card $\left(\left\{P_{x}^{\omega} \mid x \in X\right\}\right)$. To each $a \in \Gamma$ assign an element $x_{a}$ in $X$ such that $P_{x_{a}}^{\omega} \neq P_{x_{\beta}}^{\omega}$ whenever $a \neq \beta$. To simplify notation we let $P_{a}=P_{x_{\alpha}}^{\omega}$ and $P_{a}^{k}=P_{x_{a}}^{k}$ for $k \in N$ throughout the remaining part of this paper.

2.7 Lemma. If $\left\{\Theta_{n} \mid n \in N\right\}$ is a refining sequence $\bmod k$ for $X$, then for eacb $x \in X$ and each $n \in N$ there is a neigbborbood $U$ of $x$ such that $P_{z}^{1} \subseteq$ St $\left(x, \varrho_{n}\right)$ for each $z \in U \cap P_{x^{\circ}}^{1}$.

Proof. Suppose the lemma is false; then for each neighborhood $U$ of $x$ there exists $z_{U} \in U \cap P_{x}^{1}$ such that $P_{x U}^{1}-$ St $\left(x, \varrho_{n}\right) \neq \varnothing$ for some fixed $n \in N$. Let $y_{U} \in P_{z_{U}}^{1}-\mathrm{St}\left(x, \varrho_{n}\right)$; then $\left\{y_{U} \mid U\right.$ is a neighborhood of $\left.x\right\}$ is a net contained in $\bigcup\left\{P_{z}^{1} \mid z \in P_{x}^{1}\right\}$. By Lemma 2.5

$$
\bigcup\left\{P_{z}^{1} \mid z \in P_{x}^{1}\right\}=P_{x}^{2}=\bigcap_{n=1}^{\infty} \operatorname{St}\left(P_{x}^{1}, \varrho_{n}\right) .
$$

Thus the net $\left\{y_{U} \mid U\right.$ is a neighborhood of $\left.x\right\}$ has a cluster point $y$ in $P_{x}^{2}$ since $P_{x}^{2}$ is compact and so there is a subnet $\left\{y_{U_{\alpha}} \mid \alpha \in D\right\}$ of $\left\{y_{U} \mid U\right.$ is a neighborhood of $x\}$ which converges to $y$. Since $z_{U}{ }^{a} \in U$ for each neighborhood of $x$ it follows that $\left\{z_{U} \mid U\right.$ is a neighborhood of $\left.x\right\}$ converges to $x$ and thus its subnet $\left\{z_{U_{\alpha}} \mid \alpha \in D\right\}$ converges to $x$.

Observe that $x \notin P_{y}^{1}$ : If $x \in P_{y}^{1}$ then $y \in P_{x}^{1}$ and thus $\mathrm{St}\left(x, \mathcal{S}_{n}\right)$ is a neighborhood of $y$. So there exists $a \in D$ such that $y_{U_{\alpha}} \in \mathrm{St}\left(x, \varrho_{n}\right)$ contrary to the assumption that $y_{U_{\alpha}} \in P_{z_{U_{a}}}^{1}-\mathrm{St}\left(x, \varrho_{n}\right)$.

Recall that $P_{y}^{1}$ is compact; thus

$$
\bigcap_{\beta \in D}\left(\left\{z_{U_{\alpha}} \mid a \geq \beta\right\}\right)-\cap P_{y}^{1}=\{x\} \cap P_{y}^{1}=\varnothing
$$

implies there exists $\beta_{1}, \beta_{2}, \ldots, \beta_{k}$ for which $\bigcap_{i=1}^{k}\left(\left\{z_{U_{a}} \mid a \geq \beta_{i}, a \in D\right\}\right)^{-}$ $\cap P_{y}^{1}=\varnothing$. Let $\beta \in D$ such that $\beta \geq \beta_{i}$ for each $i=1,2, a, k$; then

$$
\bigcap_{i=1}^{k}\left(\left\{x_{U_{\alpha}} \mid \alpha \geq \beta_{i}, a \in D\right\}\right) \supseteq\left\{z_{U_{\alpha}} \mid \alpha \geq \beta, a \in D\right\}
$$

and so $\left(\left\{z_{U_{a}} \mid \alpha \geq \beta, \alpha \in D\right\}\right)^{-} \cap P_{y}^{1}=\varnothing$. Hence there exists $j \in N$ such that 
$\operatorname{St}\left(y, \varrho_{j}\right) \subseteq X-\left(\left\{z_{U_{\alpha}} \mid \alpha \geq \beta, \alpha \in D\right\}\right)^{-}$, that is $\operatorname{St}\left(y, \varrho_{j}\right) \cap\left(\left\{z_{U_{\alpha}} \mid a \geq \beta, \alpha \in D\right\}\right)^{-}=\varnothing$. But $\left\{z_{U_{\alpha}} \mid \alpha \geq \beta, a \in D\right\} \subseteq P_{x}^{1}$ by construction. So $\left\{z_{U_{\alpha}} \mid a \geq \beta, \alpha \in D\right\}^{-}$is a compact subset of $P_{x^{\circ}}^{1}$. Moreover

$$
\begin{aligned}
& \bigcap_{k=1}^{\infty} \operatorname{St}\left(\left(\left\{z_{U_{a}} \mid \alpha \geq \beta, \alpha \in D\right\}\right)-, \rho_{\mathcal{O}_{k}}\right)=\bigcup\left\{P_{w}^{1} \mid w \in\left(\left\{z_{U_{\alpha}} \mid \alpha \geq \beta, \alpha \in D\right\}\right)^{-}\right\} \\
& \supseteq\left(\bigcup\left\{P_{z_{U_{\alpha}}} \mid \alpha \geq \beta, \alpha \in D\right\}\right)^{-} \supseteq\left(\left\{y_{z_{U_{\alpha}}} \mid \alpha \geq \beta, \alpha \in D\right\}\right)^{-} \supseteq\{y\} .
\end{aligned}
$$

Thus $y \in S_{t}\left(\left(\left\{z_{U_{a}} \mid a \geq \beta, a \in D\right\}\right)^{-}, \varrho_{j}\right)$, a contradiction. Thus our original assumption must be false and the conclusion of the lemma follows.

2.8 Lemma. If $\left\{\varrho_{n} \mid n \in N\right\}$ is a refining sequence $\bmod k$ for $X$ and if $\varrho_{n}^{2}=\left\{S_{t}\left(x, \varrho_{n}\right) \mid x \in X\right\}$ for eacb $n \in N$, then $\left\{\Theta_{n}^{2} \mid n \in N\right\}$ is a refining sequence $\bmod k$ and moreover $\bigcap_{n=1}^{\infty}$ St $\left(C, \varrho_{n}^{2}\right)^{-}=P_{C}^{2}$ for each compact subset $C$ of $X$.

Proof. Recall that $P_{C}^{2}=\bigcup\left\{P_{z}^{1} \mid z \in C\right\}$. Clearly

$$
\begin{aligned}
\operatorname{st}\left(C, \mathscr{O}_{n}^{2}\right) & =\bigcup\left\{\operatorname{St}\left(z, \mathscr{G}_{n}\right) \mid \operatorname{St}\left(z, \varrho_{n}\right) \cap C \neq \varnothing\right\}=\bigcup\left\{\operatorname{St}\left(z, \Theta_{n}\right) \mid z \in \operatorname{St}\left(C, \Theta_{n}\right)\right\} \\
& \supseteq \bigcup\left\{P_{z}^{1} \mid z \in P_{C}^{1}\right\}=P_{C}^{2} \text { for each } n
\end{aligned}
$$

and so $\bigcap_{n=1}^{\infty}$ st $\left(C, \varrho_{n}^{2}\right)-\supseteq P_{C}^{2}$. We now show that $\bigcap_{n=1}^{\infty}$ st $\left(C, \varrho_{n}^{2}\right)^{-} \subseteq P_{C}^{2}$. Let $z \in \bigcap_{n=1}^{\infty} S_{t}\left(C, \varrho_{n}^{2}\right)^{-}$and let $U$ be an arbitrary closed neighborhood of $z$. Since $z \in \mathrm{St}\left(C, \varrho_{n}^{2}\right)^{-}$it follows that St $\left(C, \varrho_{n}^{2}\right) \cap U \neq \varnothing$ for each $n \in N$; let $z_{n} \epsilon$ St $\left(C, \mathscr{S}_{n}^{2}\right) \cap U$. So there exists $x_{n} \in X$ such that $z_{n} \in \mathrm{St}\left(x_{n}, \mathscr{G}_{n}\right)$ and St $\left(x_{n}, \Theta_{n}\right) \cap C \neq \varnothing$. Thus $x_{n} \in \mathrm{St}^{n}\left(C, \varrho_{n}\right)$ for each $n$ and thus $\left\{x_{n} \mid n \in N\right\} \cup P_{C}^{1}$ is compact by Lemma 2.3; moreover every cluster point of $\left\{x_{n} \mid n \in N\right\}$ is in $P_{C}^{1}$. Thus by Lemma $2.4\left\{z_{k} \mid k \in N\right\}$ has a cluster point $w$ in $\bigcap_{n=1}^{\infty} \operatorname{St}\left(P_{C}^{1}, \varrho_{n}\right)=P_{C}^{2}$. Clearly $w \in U$ since $U$ is closed and $U \supseteq\left\{z_{k} \mid k \in N\right\}$ and hence $z \in P_{C}^{2}$ since $U$ was an arbitrary closed neighborhood of $z$. Thus

$$
\bigcap_{n=1}^{\infty} \operatorname{st}\left(C, \varrho_{n}^{2}\right)-\subseteq P_{C}^{2} \subseteq \bigcap_{n=1}^{\infty} \operatorname{st}\left(C, \varrho_{n}^{2}\right)
$$

and so it follows that

$$
\bigcap_{n=1}^{\infty} \operatorname{st}\left(C, \Theta_{n}^{2}\right)^{-}=P_{C}^{2}=\bigcap_{n=1}^{\infty} \operatorname{st}\left(C, \Theta_{n}^{2}\right) \text {. }
$$

It remains to show that $\left\{\mathrm{St}\left(C, \mathcal{G}_{n}^{2}\right)-\mid n \in N\right\}$ is a base for the neighborhood system of $P_{C}^{2}$. Since $P_{C}^{2}$ is compact and $X$ is regular it suffices to show that $\left\{S_{t}\left(C, \varrho_{n}^{2}\right) \mid n \in N\right\}$ is a base for the neighborhood system of $P_{C}^{2}$. Let $U$ be an open neighborhood of $P_{C}^{2}$. If St $\left(C, \mathcal{G}_{n}^{2}\right) \nsubseteq U$ for any $n \in N$ then there exists $z_{n} \in \mathrm{St}\left(C, \Theta_{n}^{2}\right)-U$ for each $n \in N$. It can be shown in precisely the same way 
as was done above that the sequence $\left\{z_{n} \mid n \in N\right\}$ has a cluster point in $P_{C}^{2}$ contrary to the assumption that $z_{n} \notin U$ for any $n \in N$. So there must exist $n \in N$ such that $\mathrm{St}\left(C, \varrho_{n}\right) \subseteq U$ and this completes the proof of the lemma.

Finally we prove a result which is fundamental in proof that a space with a refining sequence mod $k$ is normal and in the proof that it is paracompace.

2.9 Lemma. If $X$ is a space with a refining sequence $\left\{\varrho_{n} \mid n \in N\right\} \bmod k$ and if $D$ is a closed discrete collection in $X$, then for each $x \in X$ and $k \in N$ there exist $n(x, k) \in N$ and a finite subset $\mathscr{D}(x, k)$ of $\mathscr{D}$ such that St $\left(P_{x}^{k}, \mathcal{S}_{n(x, k)}\right) \cap S_{t}\left(E_{x}^{k}, \mathcal{S}_{n(x, k)}\right)=\varnothing$ where $E_{x}^{k}=\bigcup\{D \mid D \in \mathscr{D}-\mathscr{D}(x, k)\}$.

Proof. Suppose the lemma is false. Then for some $x \in X$ and some $k \in N$ there exists a sequence $\left\{D_{n} \mid n \in N\right\} \subseteq \mathcal{D}$ such that $D_{j} \neq D_{i}$ for $i \neq j$ and $S_{t}\left(P_{x}^{k}, \varrho_{j}\right) \cap S_{t}\left(D_{j}, \varrho_{j}\right) \neq \varnothing$. Let $x_{j} \in \overline{S_{t}}\left(P_{x}^{k}, \varrho_{j}\right) \cap S_{t}\left(D_{j}^{j}, \varrho_{j}\right) ;$; then by Lemma $2.3 C=$ $\left\{x_{j} \mid j \in N\right\} \cup P_{x}^{k+1}$ is compact where $P_{x}^{k+1}=\bigcap_{n=1}^{\infty}$ St $\left(P_{x}^{k}, \mathcal{S}_{j}\right)$. Moreover St $\left(C, \varrho_{j}\right) \cap D_{j} \neq \varnothing$ for any $j \in N$. Let $z_{j} \in D_{j} \cap S_{t}\left(C, \varrho_{i}\right)$; then by Lemma 2.3 $\left\{z_{j} \mid j \in N\right\} \cup P_{C}^{1}$ is compact where $P_{C}^{1}=\bigcap_{n=1}^{\infty}$ St $\left(C, \varrho_{n}\right)$. Hence $\left\{z_{j} \mid j \in N\right\}$ has a cluster point; but $z_{j} \in D_{j}, z_{j} \notin D_{i}$ for $i \neq j$ and $\left\{D_{j} \mid j \in N\right\}$ is discrete, a contradiction. Thus the conclusion of the lemma follows.

3. A space with a refining sequence of covers $\bmod k$ is subparacompact. In [6] Burke gives a number of equivalent conditions each of which characterizes subparacompactness. In the present situation only the characterization in terms of $\sigma$-discrete refinements is needed.

3.1 Definition. A space $X$ is subparacompact if and only if every open cover for $X$ has a $\sigma$-discrete closed refinement.

The following proposition gives a condition which is fairly obviously equivalent to Definition 2.1 and the proof is omitted.

3.2 Proposition. Let $X$ be a regular topological space. For any open cover $\mathcal{U}$ of $X$ let $\mathcal{U}^{*}$ be the collection of all countable unions of members of $\mathcal{U}$. Then $X$ is subparacompact if and only if $U^{*}$ bas a closed $\sigma$ discrete refinement for an arbitrary open cover $\mathcal{U}$ of $X$.

3.3 Theorem. Every $T_{2}$ topological space with a refining sequence $\bmod k$ is subparacompact.

Proof. Let $X$ be a topological space with a refining sequence $\left\{\varrho_{n} \mid n \in N\right\} \bmod k_{0}$ For each $n, k \in N$ and each $x \in X$ let $V_{n}^{k}(x)=X-\bigcup\left\{S_{t}\left(y, \varrho_{k}\right) \mid x \notin \operatorname{St}\left(y, \varrho_{n}\right)\right\}$ and let $V_{n}(x)=\bigcup_{k=1}^{\infty} V_{n}^{k}(x)$. Observe:

(1) $V_{n}(x) \subseteq$ St $\left(x, \varrho_{n}\right)$ for each $x \in X$ and each $n \in N$.

Proof. Let $z \in V_{n}(x)$ then there exists $k_{0} \in N$ such that $z \in V_{n}^{k_{0}}(x)$ and 
thus $z \notin S t\left(y, \varrho_{k_{0}}\right)$ whenever $x \notin S t\left(y, \varrho_{n}\right)$. Clearly $z \in S_{t}\left(z, \varrho_{k_{0}}\right)$; thus $x \in \operatorname{St}\left(z, \varrho_{n}\right)$ and $z \in \operatorname{St}\left(x, \mathscr{S}_{n}\right)$. Hence $V_{n}(x) \subseteq \operatorname{St}\left(x, \mathcal{S}_{n}\right)$.

(2) The set $V_{n}(x)$ is an open neighborhood of $x$ for each $n$.

Proof. Clearly $x \in V_{n}(x)$ for each $n \in N$. Let $z \in V_{n}(x)$; then there exists $k_{0} \in N$ such that $z \in V_{n}^{k_{0}}(x)$. By Lemma 2.7 there exists a neighborhood $U$ of $z$ such that if $w \in U \cap P_{z}^{1^{n}}$, then $P_{w}^{1} \subseteq S_{t}\left(z, \varrho_{k_{0}}\right)$. Since $X$ is regular we may choose $U$ to be a closed neighborhood of $z$. Suppose

$$
z \in\left(\bigcup\left\{S t\left(y, \mathcal{G}_{k}\right) \mid x \notin \mathrm{St}\left(y, \mathcal{G}_{n}\right)\right\}\right)^{-}
$$

for each $k \in N$. Then we may choose a point

$$
z_{k} \in U \cap \operatorname{St}\left(z, \varrho_{k}\right) \cap\left(U\left\{\operatorname{St}\left(y, \mathcal{G}_{k}\right) \mid x \notin \operatorname{St}\left(y, \varrho_{n}\right)\right\}\right)
$$

for each $k \in N$. Thus there is $y_{k} \in X$ such that $z_{k} \in \mathrm{St}\left(y_{k}, \mathcal{S}_{k}\right)$ and $x \notin \operatorname{St}\left(y_{k}, \varrho_{n}\right)$ for each $k \in N$. Now $z_{k} \in \operatorname{St}\left(z, \varrho_{k}\right)$ and $y_{k} \epsilon$ St $\left(\left[\left\{z_{i} \mid i \in N, i \geq k\right\}\right]^{-}, \varrho_{k}\right)$ for each $k \in N$. Hence by the first part of Lemma $2.4\left(\left\{y_{k} \mid k \in N\right\}\right)^{-}$is compact. Let $y$ be a cluster point of $\left\{y_{k} \mid k \in N\right\}$ then by the second part of Lemma $2.4 y$ is in $P_{w}^{1}$ where $w$ is a cluster point of $\left\{z_{i} \mid i \in N\right\}$. By Lemma $2.3 w \in P_{z}^{1}$ and because $z_{i} \in U$ for each $i$ and $U$ is closed, $w \in U$. Hence it follows that $y \in P_{w}^{1} \subseteq S_{t}\left(z, \mathscr{G}_{k_{0}}\right)$ by the choice of $U$. Thus there is $k \in N$ such that $y_{k} \in \mathrm{St}\left(z, \varrho_{k_{0}}\right)$ since $y$ is a cluster point of $\left\{y_{k} \mid k \in N\right\}$. Recall that $x \notin \operatorname{St}\left(y_{k}, \varrho_{n}\right)$ and that $z \in V_{n}^{k_{0}}(x)$; thus $z \notin \operatorname{St}\left(y_{k}, \varrho_{k_{0}}\right)$ and hence $y_{k} \notin \mathrm{St}\left(z, \varrho_{k_{0}}\right)$, a contradiction. Hence there exists $k \in N$ such that $z \notin\left(\bigcup\left\{S_{t}\left(y, \mathscr{S}_{k}\right) \mid x \notin \mathrm{St}\left(y, \mathcal{G}_{n}\right)\right\}\right)^{-}$and so

$$
z \in X-\left(\bigcup\left\{S t\left(y, \Theta_{k}\right) \mid x \notin \operatorname{St}\left(y, \Theta_{n}\right)\right\}\right)^{-} \subseteq V_{n}^{k}(x) \subseteq V_{n}(x) \text {. }
$$

Thus it follows that $V_{n}(x)$ is an open set.

Let $<$ be a well order relation on $X$ and let $V_{n}^{k}(x)^{*}=V_{n}^{k}(x)-U\left\{V_{n}(y) \mid y<x\right\}$. If $\mathcal{C}_{n}^{k}=\left\{V_{n}^{k}(x)^{*} \mid x \in X\right\}$ then it is clear that $\mathcal{O}_{n}^{k}$ is a disjoint collection of closed sets such that $\bigcup_{k=1}^{\infty} \mathcal{O}_{n}^{k}$ covers $X$ for each $n \in \stackrel{n}{N}$. We show that $\mathcal{C}_{n}^{k}$ is closure preserving and hence discrete for each $k$ and $n \in N$. Let $A \subseteq X$ and suppose $x \in\left(\bigcup\left\{V_{n}^{k}(y)^{*} \mid y \in A\right\}\right)^{-}$. Since $P_{x}^{1}$ is compact and $S_{t}\left(x, \mathcal{G}_{k}^{-}\right) \supseteq P_{x}^{1}$ there are $G_{1}, \ldots, G_{p}^{n} \in \mathcal{G}_{k}$ such that $x \in G_{i}$ for $i=1,2, \ldots, p$ and $\bigcup_{i=1}^{p} G_{i}^{x} \supseteq P_{x}^{1}$. In addition there is $j \geq k$ such that $\operatorname{St}\left(x, \varrho_{j}\right) \subseteq \bigcup_{i=1}^{p} G_{i}$. Let $w$ be the first element in $X$ such that $x \in V_{n}(w)$. Then for each $y \in A, y<w$ there exists $z(y) \in X$ such that $x \in \mathrm{St}\left(z(y), \mathcal{S}_{j}\right)$ and $y \notin \mathrm{St}\left(z(y), \mathcal{S}_{n}\right)$. In particular note that $z(y) \in \mathrm{St}_{\mathrm{t}}\left(x, \varrho_{j}\right)$ and thus $z(y) \in G_{i}$ for some $i=1,2, \ldots, p$ and for each $y \in A$ where $y<w$. Let $W_{i}=\left(\bigcup\left\{V_{n}^{k}(y)^{*} \mid y \in A, y<w \text { and } z(y) \in G_{i}\right\}\right)^{-}$for $i=1,2, \cdots, p$. Let $W_{0}=\left(\bigcup\left\{V_{n}^{k}(y)^{*} \mid y \in A \text { and } y>w\right\}\right)^{-}$. Then 


$$
\left(\bigcup\left\{V_{n}^{k}(y)^{*} \mid y \in A\right\}\right)^{-} \subseteq\left(\bigcup_{i=0}^{p} w_{i}\right) \cup V_{n}^{k}(w)^{*} .
$$

Thus $x \in W_{i}$ for some $i=0,1,2, \ldots, p$ or $x \in V_{n}^{k}(w)^{*}$ and $w \in A$. Clearly $x \notin W_{0}$ since $x$ is in the open set $V_{n}(w)$ and $V_{n}(w) \cap\left(U\left\{V_{n}^{k}(y)^{*} \mid y>w\right\}\right)=\varnothing$. If $x \in W_{i}$ for some $i=1,2, \cdots, p$, then $G_{i} \cap\left(\bigcup\left\{V_{n}^{k}(y)^{*} \mid y \in A, y<w\right.\right.$, and $\left.\left.z(y) \in G_{i}\right\}\right) \neq \varnothing$ since $x \in G_{i}$. Let $z \in G_{i} \cap\left(\bigcup\left\{V_{n}^{k}(y)^{*} \mid y \in A, y<w\right.\right.$ and $\left.\left.z(y) \in G_{i}\right\}\right)$. Then there is $y \in A, y<w$ such that $z \in V_{n}^{k}(y)^{*}$ and $z(y) \in G_{i}$. Moreover $z \in G_{i}$; so $z \in \mathrm{St}\left(z(y), \varrho_{k}\right)$; but recall $y \notin \mathrm{St}\left(z(y), \varrho_{n}\right)$. Thus $z \notin V_{n}^{k}(y)^{*}$, a contradiciton. So $x \notin W_{i}$ for $i=0,1,2, \ldots, p$. Hence $x \in V_{n}^{k}(w)^{*}$ and $w \in A$; therefore $\left(\bigcup\left\{V_{n}^{k}(y)^{*} \mid y \in A\right\}\right)^{-}=\bigcup\left\{V_{n}^{k}(y)^{*} \mid y \in A\right\}$ and hence $\bigcirc_{n}^{k}$ is discrete.

Finally let $\mathcal{U}$ be any open cover for $X$ and let $\mathcal{U}^{*}$ be the set of all countable unions of members of $\mathcal{U}$. Then for each $x \in X$ there is $U^{*} \epsilon \mathcal{U}^{*}$ such that $P_{x}^{2} \subseteq U^{*}$ since $P_{x}^{2}$ is compact. By Lemma 2.8 there is $n \in N$ such that St $\left(x, \varrho_{n}^{2}\right)$ $=\bigcup\left\{S_{t}\left(y, \mathscr{G}_{n}\right) \mid x \in \mathrm{St}\left(y, \mathscr{G}_{n}\right)\right\} \subseteq U^{*}$. Now there is $k \in N$ and $y \in X$ such that $x \in V_{n}^{k}(y)^{*}$; but $V_{n}^{k}(y)^{*} \subseteq \operatorname{St}\left(y, \mathcal{Q}_{n}\right)$ and $\operatorname{St}\left(y, \varrho_{n}\right) \subseteq U^{*}$ since $x \in \operatorname{St}\left(y, \varrho_{n}\right)$. Thus $x \in V_{n}^{k}(y)^{*} \subseteq U^{*}$. It follows that $\bigcup_{k=1}^{\infty} \bigcup_{n=1}^{\infty} \mathcal{U}_{n}^{k}(\mathcal{U})$ is a closed $\sigma$-discrete refinement of $\mathcal{U}^{*}$ where $\mathcal{U}_{n}^{k}(\mathcal{U})=\left\{V_{n}^{k}(y)^{*} \in \mathcal{C}_{n}^{k} \mid V_{n}^{k}(y)^{*} \subseteq U^{*}\right.$ for some $U^{*} \epsilon \mathcal{U}^{*}$ for each $k$ and $n \in N\}$. Hence by Proposition $3.2 X$ is subparacompact.

4. A space with a refining sequence $\bmod k$ is normal. In the proof that a space with a refining sequence $\bmod k$ is normal the fact that such a space is subparacompact is needed and in addition the proof relies heavily on Lemma 2.9 and the following lemma.

4.1 Lemma. Let $A$ and $B$ be a closed disjoint subsets of $X$. Then there exists an open cover $\mathcal{U}$ for $X$ such that if $U \in \mathcal{U}$ and $U \cap A \neq \varnothing$ then $U^{-} \cap B$ or if $U \cap B \neq \varnothing$ then $U^{-} \cap A=\varnothing$.

Proof. For each $x \in A$ there exist open sets $U_{x}$ and $U(B)$ such that $x \in U_{x}, B \subseteq U(B)$ and $U_{x} \cap U(B)=\varnothing$. Similarly for each $x \in B$ there exist open sets $U_{x}$ and $U(A)$ such that $x \in U_{x}, A \subseteq U(A)$ and $U_{x} \cap U(A)=\varnothing$. Let

$$
\mathcal{U}=\left\{U_{x} \mid x \in A \cup B\right\} \cup\{X-A \cap X-B\}
$$

If $U_{x} \in \mathcal{U}$ such that $U_{x} \cap A \neq \varnothing$ then $x \in A$ and clearly $U_{x}^{-} \cap B=\varnothing$. Similarly if $U_{x} \cap B \neq \varnothing$ then $x \in B$ and $U_{x}^{-} \cap A=\varnothing$. Thus $\mathcal{U}$ is the desired open cover.

4.2 Theorem. $A T_{2}$ space $X$ with a refining sequence $\bmod k$ is normal. 
Proof. Let $A$ and $B$ be disjoint closed subsets of $X$ and let $U$ be an open cover for $X$ satisfying Lemma 4.1 relative to $A$ and $B$. Since $X$ is subparacompact there exists a $\sigma$-discrete closed refinement $\mathscr{D}=\bigcup_{m=1}^{\infty} \mathscr{D}_{n}$ for $\mathcal{U}$.

Let $n$ be a fixed natural number and let $A_{n}=A \cap\left(\bigcup\left\{D \mid D \in D_{n}\right\}\right)$. For each $D \in \mathbb{D}_{n}$ let $U(D) \in \mathcal{U}$ such that $D \subseteq U(D)$. By Lemmas 2.8 and 2.9 for each $x \in X$ there exist $n_{x}$ and a finite subset $\mathscr{D}(n, x)$ of $\mathscr{D}_{n}$ such that $\mathrm{St}\left(x, \mathcal{G}_{n_{x}}^{2}\right) \cap$ St $\left(E_{x}^{n}, \varrho_{n_{x}}^{2}\right)=\varnothing$ where $E_{x}^{n}=\bigcup\left\{D \mid D \in \mathscr{D}_{n}-\mathcal{D}(n, x)\right\}_{\text {. If } x \in B \text { let }}$

$$
W_{x}=\operatorname{St}\left(x, G_{n_{x}}\right)-(\bigcup\{U(D) \mid D \in \mathscr{D}(n, x) \text { and } D \cap A \neq \varnothing\})-
$$

By Lemma $4.1 U(D)^{-} \cap B=\varnothing$ for any $D \in \mathscr{D}_{n}$ such that $D \cap A \neq \varnothing$. Thus $(\bigcup\{U(D) \mid D \in \mathscr{D}(n, x) \text { and } D \cap A \neq \varnothing\})^{-} \cap B=\varnothing$ since $\mathscr{D}(n, x)$ is a finite set and so $W_{x}$ is an open neighborhood of $x$. Let $W_{k}=\bigcup\left\{W_{x} \mid x \in B\right.$ and $\left.n_{x}=k\right\}$. Clearly $W_{k}$ is an open set and $\bigcup_{k=1}^{\infty} W_{k} \supseteq B$. Moreover $W_{k}^{-} \cap A_{n}=\varnothing$ for any $k \in N$. For suppose $z \in W_{k}^{-} \cap A_{n}$; then there exists $D_{z} \in \mathscr{D}_{n}$ such that $z \in D_{z^{*}}$ Let $G \in \Theta_{k}$ such that $z \in G$; then $G \cap U\left(D_{z}\right) \cap W_{k} \neq \varnothing$ since $z \in W_{k}^{-}$. Let $y \in G \cap U\left(D_{z}\right) \cap W_{k}$; then there exists $x \in B$ such that $n_{x}=k$ and $y \in W_{x} \subseteq$ St $\left(x, \varrho_{n_{x}}\right)$. Hence it follows that $z \in \mathrm{St}\left(x, \varrho_{n_{x}}^{2}\right)$. So $D_{z} \in \mathscr{D}(n, x)$ since St $\left(x, \varrho_{n_{x}}^{n_{x}}\right) \cap S_{t}\left(D, \varrho_{n_{x}}^{2}\right)=\varnothing$ if $D \in \mathscr{D}_{n}-\mathscr{D}(n, x)$ and hence $w_{x} \subseteq X-U\left(D_{z}\right)$. But $y \in U\left(D_{z}\right) \cap W_{x}$, a contradiction. Thus it follows that for any $k \in N, W_{k}^{-} \cap A_{n}=\varnothing$. Now if $x \in A_{n}$ let $U_{x}=\operatorname{St}\left(x, \Theta_{n_{x}}\right) \cap U\left(D_{x}\right)$ where $D_{x} \in \mathscr{D}_{n}$ such that $x \in D_{x^{\circ}}$. Let $U(m, n)=\bigcup\left\{U_{x} \mid x \in A_{n}\right.$ and $\left.n_{x}=m\right\}$; clearly $U(m, n)$ is an open set and $\bigcup_{m=1}^{\infty} U(m, n) \supseteq A_{n}$. Moreover $U(m, n)-\cap B=\varnothing$ for any $m \in N$. For suppose $z \in U(m, n)^{-} \cap B$; then there exists $G \in \oint_{m}$ such that $z \in G$ and $G \cap U(m, n)$ $\neq \varnothing$; let $y \in G \cap U(m, n)$. Then there exists $x \in A_{n}$ such that $n_{x}=m$ and $y \in U_{x} \subseteq S_{t}\left(x, \varrho_{n_{x}}\right)$. Thus it follows that $z \in S_{t}\left(x, \varrho_{n_{x}}^{2}\right)$. Note that if

$$
s \in U(m, n)-(\bigcup\{U(D) \mid D \cap A \neq \varnothing \text { and } D \in \mathscr{D}(n, x)\})-
$$

then there exist $w \in A_{n}$ and $D_{w} \in \mathscr{D}_{n}$ such that $n_{w}=m, w \in D_{w} \cap A$ and $s \in U_{w}=S_{t}\left(w, \mathscr{S}_{n_{w}}\right) \cap U\left(D_{w}\right)$. Thus since $s \in U\left(D_{w}\right)$ and $s \notin \bigcup\{U(D) \mid D \cap$ $A \neq \varnothing$ and $D \in \mathscr{T}(n, x)\}$ it follows that $D_{w} \notin \mathscr{D}(n, x)$. So

$$
\begin{aligned}
& U(m, n)-(\bigcup\{U(D) \mid D \cap A \neq \varnothing \text { and } D \in \mathscr{D}(n, x)\})- \\
& \subseteq U(m, n)-\bigcup\{U(D) \mid D \cap A \neq \varnothing \text { and } D \in \mathscr{D}(n, x)\} \\
& =\bigcup\left\{U_{w} \mid w \in A_{n} \text { and } n_{w}=m\right\}-\bigcup\{U(D) \mid D \cap A \neq \varnothing \text { and } D \in \mathscr{D}(n, x)\} \\
& \subseteq \bigcup\left\{\operatorname{St}\left(w, \mathscr{S}_{n_{w}}\right) \mid w \in D \cap A, D \in \mathscr{D}_{n}-\mathscr{D}(n, x) \text { and } n_{w}=n_{x}=m\right\} \\
& \subseteq \bigcup\left\{S_{t}\left(D, \mathscr{S}_{n_{x}}^{2}\right) \mid D \in \mathscr{D}_{n}-\mathscr{D}(n, x)\right\}=\operatorname{St}\left(E_{x^{\prime}}^{n}, \mathscr{S}_{n_{x}}^{2}\right) \text {. }
\end{aligned}
$$


Now recall that $S_{t}\left(x, \varrho_{n_{x}}^{2}\right) \cap S_{t}\left(E_{x}^{n}, \varrho_{n_{x}}^{2}\right)=\varnothing$ and that

$$
(\bigcup\{U(D) \mid D \cap A \neq \varnothing \text { and } D \in \mathscr{D}(n, x)\})^{-} \cap B=\varnothing \text {. }
$$

So that

$$
O_{z}=\operatorname{St}\left(x, \mathcal{O}_{n_{x}}^{2}\right) \cap\left(X-(U\{U(D) \mid D \cap A \neq \varnothing \text { and } D \in \mathscr{D}(n, x)\})^{-}\right)
$$

is an open neighborhood of $z$ and $O_{z} \cap U(m, n)=\varnothing$, contradicting the assumption that $z \in U(m, n)^{-} \cap B$. Hence $U(m, n)^{-} \cap B=\varnothing$ for any $m \in N$, Finally for the fixed natural number $n$ let

$$
U_{n}=\bigcup_{m=1}^{\infty}\left[U(m, n)-\left(\bigcup_{i=1}^{n} W_{i}\right)^{-}\right] \text {and } W_{n}=\bigcup_{m=1}^{\infty}\left[W_{m}-\left(\bigcup_{i=1}^{n} U(i, n)\right)^{-}\right] \text {. }
$$

Since $W_{i}^{-} \cap A_{n}=\varnothing$ and $U(i, n)^{-} \cap B=\varnothing$ for each $i \in N$, it follows that $U_{n} \supseteq A_{n}, W_{n} \supseteq B$ and $U_{n} \cap W_{n}=\varnothing$. So in particular $U_{n}^{-} \cap B=\varnothing$.

Thus since $n$ was an arbitrary member of $N$, it follows that we can construct a sequence $\left\{U_{n} \mid n \in N\right\}$. of open sets such that $U_{n} \supseteq A_{n}$ and $U_{n}^{-} \cap B=\varnothing$ for any $n \in N$.

By a dual argument we can construct a sequence $\left\{V_{n} \mid n \in N\right\}$ of open sets such that $V_{n} \supseteq B_{n}=B \cap\left(\bigcup\left\{D \mid D \in D_{n}\right\}\right)$ and $V_{n}^{-} \cap A=\varnothing$ for each $n \in N$.

Thus if we let

$$
U=\bigcup_{n=1}^{\infty}\left[U_{n}-\left(\bigcup_{i=1}^{n} V_{i}\right)^{-}\right] \text {and } V=\bigcup_{n=1}^{\infty}\left[V_{n}-\left(\bigcup_{i=1}^{n} U_{i}\right)^{-}\right] \text {, }
$$

then $U$ and $V$ are open sets such that $U \supseteq A, V \supseteq B$ and $U \cap V=\varnothing$. It follows that $X$ is a normal topological space.

5. The Proof of Theorem 1.3. Having established that a $T_{2}$ space $X$ with a refining sequence $\bmod k$ is normal it is clear that $X$ is a $p$-space. More precisely we have

5.1 Lemma. If $X$ is a $T_{2}$ space then the following are equivalent:

(a) $X$ bas a refining sequence $\bmod k$.

(b) $X$ is a normal space witb a p-sequence $\left\{\mathcal{H}_{n} \mid n \in N\right\}$ in $\beta X$ such that $\bigcap_{n=1}^{\infty}$ St $\left(C, \mathcal{H}_{n}\right)-\bar{\beta} X=\bigcap_{n=1}^{\infty}$ St $\left(C, \mathcal{H}_{n}\right) \subseteq X$ for each compact subset $C$ of $X$.

The proof of this lemma is straightforward and is omitted.

In $\$ 2$ we defined $\Gamma$ to be the first ordinal number whose cardinal number is the cardinal number of $\left\{P_{x}^{\omega} \mid x \in X\right\}$ and for each $a \in \Gamma$ we assigned as element $x_{a}$ in $X$ such that $x_{a} \notin P_{x \beta}^{\omega}$ if $a \neq \beta$. We also defined $P_{a}^{k}=P_{x_{a}}^{k}$ for each $k \in N$ and $P_{\alpha}=P_{x_{a}}^{\omega}$. This terminology will be used below. 
If $\mathscr{D}$ is a discrete set in a space with a refining sequence $\left\{\mathscr{G}_{n} \mid n \in N\right\} \bmod k$ then by Lemma 2.9 there exists $n(\alpha, k) \in N$ and a finite subset $\mathscr{D}(\alpha, k)$ of $\mathfrak{D}$ such that $S_{t}\left(P_{a}^{k}, \mathscr{S}_{n(a, k)}\right) \cap S_{t}\left(E_{a}^{k}, \varrho_{n(a, k)}\right)=\varnothing$ for each $\alpha \in \Gamma$ and each $k \in N$. Recall that $E_{a}^{k}=\bigcup\{D \mid D \in \mathcal{D}-\mathscr{D}(\alpha, k)\}$. Let $\Gamma(k, j)=\{\alpha \in \Gamma \mid n(\alpha, k)=j\}$ and let $B(k, j, D)=\bigcup\left\{P_{a}^{k} \cap D \mid \alpha \in \Gamma(k, j)\right\}$ for each $D \in \mathcal{D}$. We then have:

5.2 Lemma. If $X$ is a $T_{2}$ space with a refining sequence $\bmod k$ and $D$ is a closed discrete collection of subsets of $X$, then there exists a $\sigma$-discrete collection $\mathcal{O}=\bigcup_{k=1}^{\infty} \bigcup_{j=1}^{\infty} \mathcal{Q}(k, j)$ of open subsets of $X$ with the property that for eacb $B(k, j, D)$ (as defined above) there is $V(k, j, D) \in \mathcal{Q}(k, j)$ such that $B(k, j, D) \subseteq V(k, j, D)$.

Proof. Let $\Gamma(k, j)$ be defined as above and let $\alpha_{0}$ be the first member of $\Gamma(k, j)$. Since $\mathcal{D}\left(a_{0}, k\right)$ is a finite collection of disjoint closed sets and $X$ is normal by Theorem 4.2, there exist open sets $O(D)$ for each $D \in \mathscr{D}\left(a_{0}, k\right)$ such that $D \subseteq O(D)$ and $O(D) \cap O\left(D^{*}\right)=\varnothing$ whenever $D^{*} \neq D, D^{*}$ and $D \in \mathscr{D}\left(a_{0}, k\right)$. Assume for $\beta>a_{0}, \beta \in \Gamma(k, j)$ that $O(D)$ has been defined for each $D \in \mathscr{D}(a, k)$ where $\alpha \in \Gamma(k, j)$ and $\alpha<\beta$. If $\mathscr{D}(\beta, k)-\bigcup\left\{D^{\prime} \mid D^{\prime} \in \mathscr{D}(\alpha, k), \alpha \in \Gamma(k, j)\right.$ and $a<\beta\}=\varnothing$ then there is no problem. If this set is not empty let $D$ be any mem. ber of it and let $O(D)$ be an open set such that

(a) $O(D) \supseteq D$,

(b) $O(D)^{-} \cap\left(\bigcup\left\{D^{*} \mid D^{*} \in D(\alpha, k), \alpha \in \Gamma(k, j)\right.\right.$ and $\left.\left.a<\beta\right\}\right)=\varnothing$,

(c) $O(D) \cap O\left(D^{*}\right)=\varnothing$ if $D^{*} \neq D$ and $D^{*} \in \mathcal{D}(\beta, k)-\bigcup\left\{D^{\prime} \mid D^{\prime} \in \Gamma(a, k)\right.$, $\alpha \in \Gamma(k, j)$ and $\alpha<\beta\}$.

By induction on the well ordering of $\Gamma(k, j)$ we define an open set $O(D)$ satisfying (a), (b), and (c) above for each $D \in \mathscr{D}(\alpha, k)$ and each $\alpha \in \Gamma(k, j)$.

Let $a \in \Gamma(k, j)$; let $a_{1}$ be the first member of $\Gamma(k, j)$ such that $\mathcal{D}(\alpha, k) \cap$ $\mathscr{D}\left(a_{1}, k\right) \neq \varnothing$ and let $\mathscr{D}(a, k) \cap \mathscr{D}\left(a_{1}, k\right)=\mathscr{D}_{1}(a, k)$. Let $\alpha_{2}$ be the first member of $\Gamma(k, j)$ such that $\left[\mathscr{D}(a, k)-\mathscr{D}\left(a_{1}, k\right)\right] \cap \mathscr{D}\left(a_{2}, k\right) \neq \varnothing$ and let $\mathscr{D}_{2}(a, k)=$ $\left[\mathscr{D}(a, k)-\mathscr{D}\left(a_{1}, k\right)\right] \cap \mathscr{D}\left(a_{2}, k\right)$. Continue this process until $\mathscr{D}(\alpha, k)-$ $\bigcup_{i=1}^{n} D_{i}(a, k)=\varnothing$. Note that this process must terminate after a finite number of steps since $\mathscr{D}(a, k)$ is finite and note that $a_{1}<a_{2}<\ldots<a_{n^{*}}$. For each $i$ and each $D \in \mathscr{D}_{i}(\alpha, k)$ let

$$
\alpha(D, \alpha)=\left[O(D)-\left(\bigcup\left\{O\left(D^{\prime}\right) \mid D^{\prime} \in \mathfrak{D}_{m}(\alpha, k) \text { and } m>i\right\}\right)^{-}\right] \cap \operatorname{St}\left(P_{a}^{k} \cap D, \mathscr{S}_{j}\right) \text {. }
$$

Note that if $m>i$ then $a_{m}>\alpha_{i}$ and thus $O\left(D^{\prime}\right)^{-} \cap D \in \mathscr{D}_{m}(\alpha, k)=\varnothing$ by condition (b) above. Thus

$$
\begin{aligned}
\left(U\left\{\alpha\left(D^{\prime}\right) \mid D^{\prime} \in \mathcal{D}_{m}(\alpha, k), m>i\right\}\right)^{-} \cap D \\
=\bigcup\left\{\left(D^{\prime}\right)-\mid D^{\prime} \in \mathcal{D}_{m}(\alpha, k), m>i\right\} \cap D=\varnothing
\end{aligned}
$$


and so $\left.O(D, a) \supseteq \mathrm{St}_{(} P_{a}^{k} \cap D, \mathcal{S}_{j}\right) \cap D$. For each $D \in \mathfrak{D}$, let $W(k, j, D)=$ $\bigcup\left\{O(D, a) \mid \alpha \epsilon \Gamma(k, j)\right.$ and $\left.P_{\alpha}^{k} \cap D \neq \varnothing\right\}$ if there is some $a \in \Gamma(k, j)$ such that $P_{a}^{k} \cap D \neq \varnothing$ and let $W(k, j, D)=\varnothing$ otherwise. Note that $W(k, j, D) \subseteq O(D)$ since $O(D, \alpha) \subseteq O(D)$ for each $\alpha \in \Gamma(k, j)$.

We show that $B(k, j, D)^{-} \subseteq W(k, j, D)$ : Let $z \in B(k, j, D)^{-}$; then $z \epsilon$ St $\left(B(k, j, D), \varrho_{j}\right)$ for otherwise St $\left(z, \varrho_{j}\right) \cap B(k, j, D)=\varnothing$. Hence it follows that $z \in S_{t}\left(P_{a}^{k} \cap D, \varrho_{j}\right)$ for some $a \in \Gamma(k, j)$, since $B(k, j, D)=\bigcup\left\{P_{a}^{k} \cap D \mid\right.$ $\alpha \in \Gamma(k, j)\}$. Clearly $D \in \mathscr{D}(a, k)$ and thus $z \in D \cap \mathrm{St}_{\mathrm{t}}\left(P_{\alpha}^{k} \cap D, \mathcal{S}_{j}\right) \subseteq O(D, \alpha)$ since $B(k, j, D)^{-} \subseteq D$. Hence $B(k, j, D)^{-} \subseteq W(k, j, D)$. Next we show that $W(k, j, D) \cap W\left(k, j, D^{*}\right)=\varnothing$ for $D, D^{*} \in \mathscr{D}^{-}$and $D \neq D^{*}$. Suppose $z \in W(k, j, D)$ $\cap W\left(k, j, D^{*}\right)$ for some $D, D^{*} \in \mathscr{D}$ and $D \neq D^{*}$. So there exist $\alpha$, and $\beta \in \Gamma(k, j)$ such that $z \in O(D, \alpha)$ and $z \in O\left(D^{*}, \beta\right)$. Let $\alpha^{*}$ and $\beta^{*}$ be the first members of $\Gamma(k, j)$ such that $D \in \mathscr{D}\left(\alpha^{*}, k\right)$ and $D^{*} \in \mathscr{D}\left(\beta^{*}, k\right)$ respectively. Since $P_{\alpha}^{k} \cap D \neq \varnothing$ and $P_{\beta}^{k} \cap D^{*} \neq \varnothing$ we have that $D \in \mathscr{D}(\alpha, k)$ and $D^{*} \in \mathscr{D}(\beta, k)$. Thus $\alpha^{*}$ and $\beta^{*}$ both exist. Recall that $W(k, j, D) \subseteq O(D), W\left(k, j, D^{*}\right) \subseteq O\left(D^{*}\right)$ and by by condition (c) above $O(D) \cap O\left(D^{*}\right)=\varnothing$ if $\alpha^{*}=\beta^{*}$. Thus $\alpha^{*}<\beta^{*}$ or $\beta^{*}<\alpha^{*}$. It suffices to assume $\alpha^{*}<\beta^{*}$. Note that $\mathrm{St}_{\mathrm{t}}\left(D^{*}, \varrho_{j}\right) \supseteq \mathrm{St}_{\boldsymbol{1}}\left(P_{\beta}^{k} \cap D^{*}, \varrho_{j}\right) \supseteq$ $O\left(D^{*}, \beta\right)$ and St $\left(P_{\alpha}^{k}, \mathcal{G}_{j}\right) \supseteq S_{t}\left(P_{\alpha}^{k} \cap D, \varrho_{j}\right) \supseteq O(D, a)$. Thus it follows that $D^{*} \in \mathscr{D}(\alpha, k)$ since $z \in O(D, a) \cap O\left(D^{*}, \beta\right)$ and since St $\left(P_{\alpha}^{k}, \varrho_{j}\right) \cap$ St $\left(D^{\prime}, \varrho_{j}\right)$ $=\varnothing$ if $D^{\prime} \notin \mathscr{D}(\alpha, k)$. Since $D$ and $D^{*} \in \mathscr{D}(\alpha, k)$ and $\alpha^{*}<\beta^{*}$, then $D \in \mathscr{D}_{i}(\alpha, k)$, $D^{*} \in \mathfrak{D}_{m}(\alpha, k)$ and $i<m$. So by definition $O(D, \alpha) \subseteq O(D)-O\left(D^{*}\right)$ and $O\left(D^{*}\right) \supseteq O\left(D^{*}, \beta\right)$ contradicting the as sumption that $z \in O(D, \alpha) \cap O\left(D^{*}, \beta\right)$.

Thus it follows that $W(k, j, D) \cap W\left(k, j, D^{*}\right)=\varnothing$ whenever $D \neq D^{*}$.

Now recall that $B(k, j, D)^{-} \subseteq D$ for each $D \in \mathcal{D}$. Thus $\left\{B(k, j, D)^{-} \mid D \in \mathcal{D}\right\}$ is a discrete collection and since $X$ is normal there exists a discrete collection $\{V(k, j, D) \mid D \in \mathcal{D}\}$ of open sets such that $B(k, j, D)^{-} \subseteq V(k, j, D) \subseteq W(k, j, D)$. Let $\mathcal{O}(k, j)=\{V(k, j, D) \mid D \in \mathcal{D}\}$ and let $\mathcal{O}=\bigcup_{k=1}^{\infty} \bigcup_{j=1}^{\infty} \mathcal{C}(k, j)$. Clearly $\mathcal{O}$ is the desired collection.

Finally we prove Theorem 1.3.

Proof of Theorem 1.3. In [7] Filippov constructed a $p$-sequence $\left\{\mathcal{H}_{n} \mid n \in N\right\}$ for a paracompact $p$-space $X$ satisfying the condition that $\bigcap_{n=1}^{\infty}$ St $\left(C, \mathcal{H}_{n}\right)-\bar{\beta} X=$ $\bigcap_{n=1}^{\infty}$ St $\left(C, \mathcal{H}_{n}\right) \subseteq X$ for each compact subset $C$ of $X$. Thus by Lemma 5.1, a paracompact $p$-space has a refining sequence $\bmod k$.

Conversely assume $X$ is a $T_{2}$ space with a refining sequence $\left\{\Theta_{n} \mid n \in N\right\} \bmod k$. Then by Theorem $4.2 X$ is normal and hence by Lemma 5.1 is a $p$-space.

Let $\mathcal{U}$ be any open cover of $X$. Since $X$ is subparacompact by Theorem 3.2, there exists a $\sigma$-discrete closed refinement $\mathscr{D}=\bigcup_{n=1}^{\infty} \mathscr{D}_{n}$ for $X$. Thus for each $n \in N$ by Lemma 5.2 there exists a $\sigma$-discrete collection $\mathcal{C}_{n}=\bigcup_{k=1}^{\infty} \bigcup_{j=1}^{\infty} \bigotimes_{n}(k, j)$ of open sets such that for each $B_{n}(k, j, D)$ there exists $V_{n}(k, j, D) \in \mathcal{C}_{n}(k, j)$ with $B_{n}(k, j, D) \subseteq V_{n}(k, j, D)$ where $B_{n}(k, j, D)=\bigcup\left\{P_{a}^{k} \cap D \mid D \in D_{n}\right\}$. Let $x \in X$; then there exists $k, n \in N, D \in \mathscr{D}_{n}$ and $a \in \Gamma$ such that $x \in D^{n} \cap P_{\alpha}^{k}$ 
hence $x \in B_{n}(k, j, D)$. Thus $\left\{B_{n}(k, j, D) \mid D \in \mathscr{D}_{n}, n, k\right.$ and $\left.j \in N\right\}$ covers $X$.

For each $n$ and each $D \in \mathscr{D}_{n}$ let $U(D)$ be a fixed member of $\mathcal{U}$ such that $D \subseteq U(D)$. Then let $U_{n}(k, j, D)=U(D) \cap V_{n}(k, j, D)$ for each $k$ and $j \in N$. Clearly $U_{n}(k, j, D) \supseteq B_{n}(k, j, D)$. So

$$
\mathcal{U}^{*}=\bigcup_{n=1}^{\infty} \bigcup_{k=1}^{\infty} \bigcup_{j=1}^{\infty}\left\{U_{n}(k, j, D) \mid D \in D_{n}\right\}
$$

is an open refinement of $\mathcal{U}$ and moreover $\left\{U_{n}(k, j, D) \mid D \in D_{n}\right\}$ is discrete since $\mathcal{C}_{n}(k, j)$ is discrete. Thus it follows that $\mathcal{U}^{n}$ is an open $\sigma$-discrete refinement of $\mathcal{U}$. Hence $X$ is paracompact and the proof is complete.

In conclusion it is observed that if $X$ is a preimage of a metric space under a perfect map then it is fairly obvious that $X$ has a refining sequence mod $k$. However, it is not at all obvious that if $X$ is a space with a refining sequence $\left\{\bigcup_{n} \mid n \in N\right\} \bmod k$ then it is a perfect preimage of a metric space. The problem is that $\left\{P_{x}^{1} \mid x \in X\right\}$ need not partition the space $X$. Moreover if $\left\{\Theta_{n} \mid n \in N\right\}$ is refined in some way to make the resulting $\left\{P_{x}^{1} \mid x \in X\right\}$ partition $X$, as can be done in subparacompact spaces, the new sequence need not be a refining sequence $\bmod k$.

\section{BIBLIOGRAPHY}

1. P. S. Aleksandrov, On some results concerning topological spaces and their continuous mappings, General Topology and its Relations to Modern Analy sis and Algebra (Proc. Sympo s., Prague, 1961), Academic Press, New York; Publ. House Czech. Acad. Sci. Prague, 1962, pp. 41-54. MR $26 \# 3003$.

2. A. V. Arhangel'skii, On a class of spaces containing all metric and all locally bicompact spaces, Dokl. Akad. Nauk SSSR 151 (1963), 751-754 = Soviet Math. Dokl. 4 (1963), 1051-1055. MR 27 \#2959.

3. - Bicompact sets and the topology of spaces, Trudy Moskov Mat. Obšč. 13 (1965), 3-55 = Trans. Moscow Math. Soc. 1965, 1-62. MR 33 \#3251.

4. - On a class of spaces containing all metric spaces and all locally compact spaces, Mat. Sb. 67 (109) (1965), 55-88; English transl., Amer. Math. Soc. Transl. (2) 92 (1970), 1-39. MR 32 \#8299; $42 \# 3$.

5. - Mappings and spaces, Uspehi Mat. Nauk 21 (1966), no. 4 (130), 133184 = Russian Math. Survey s 21 (1966), no. 4, 115-162. MR 37 \#3534.

6. D. K. Burke, On subparacompact spaces, Proc. Amer. Math. Soc. 23 (1969), 655663. MR $40 \# 3508$.

7. V. V. Filippov, On the perfect image of a paracompact p-space, Dokl. Akad. Nauk SSSR 176 (1967), 533-535 = Soviet Math. Dokl. 8 (1967), 1151-1153. MR 36 \#5903.

8. J. L. Kelley, General topology, Van Nostrand, Princeton, N. J., 1955. MR 16, 1136.

9. K. Morita, Products of normal spaces with metric spaces, Math. Ann. 154 (1964), 365-382. MR 29 \#2773.

10. - Some properties of M-spaces, Proc. Japan Acad. 43 (1967), 869-872. MR $37 \# 3517$.

DEPARTMENT OF MATHEMATICS, SAM HOUSTON STATE UNIVERSITY, HUNTSVILLE, TEXAS 77340 